\title{
The AGN content of ultraluminous IR galaxies: High resolution VLA imaging of the IRAS 1 Jy ULIRG sample*
}

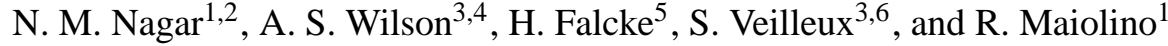 \\ 1 Arcetri Observatory, Largo E. Fermi 5, Florence 50125, Italy \\ e-mail: neil@arcetri.astro.it,maiolino@arcetri.astro.it \\ 2 Kapteyn Institute, University of Groningen, Landleven 12, 9747 AD Groningen, The Netherlands \\ e-mail: nagar@astro.rug.nl \\ 3 Department of Astronomy, University of Maryland, College Park, MD 20742, USA \\ e-mail: wilson@astro.umd.edu, veilleux@astro.umd.edu \\ 4 Adjunct Astronomer, Space Telescope Science Institute, 3700 San Martin Drive, Baltimore, MD 21218, USA \\ 5 Max-Planck-Institut für Radioastronomie, Auf dem Hügel 69, 53121 Bonn, Germany \\ e-mail: hfalcke@mpifr-bonn.mpg.de \\ ${ }^{6}$ Current address: 320-47 Downs Lab., Caltech, Pasadena, CA 91125 and Observatories of the Carnegie Institution \\ of Washington, 813 Santa Barbara Street, Pasadena, CA 91101, USA \\ e-mail: veilleux@ulirg.caltech.edu
}

Received 17 April 2003 / accepted 7 July 2003

\begin{abstract}
This paper presents the results of a high resolution radio imaging survey of 83 of the 118 ultraluminous infrared galaxies (ULIRGs) in the IRAS 1 Jy ULIRG sample. We have observed these ULIRGs at $15 \mathrm{GHz}$ with the Very Large Array (VLA). We find that $\sim 75 \%$ of Seyferts (both type 1 and 2) and LINERs have radio nuclei which are compact at our 150 mas resolution. The detection rate of $\mathrm{H}$ II nuclei is significantly lower $(32 \%)$; the detections among these are preferentially $\mathrm{HII}+$ LINER/Seyfert composite nuclei. Among ULIRGs with multiple optical or near-IR nuclei our observations detected only one (or no) nucleus; in these the radio detection is typically towards the brightest near-IR nucleus. The compactness of the radio sources, the higher detection rates in AGN-type nuclei than H II nuclei, the preferential detection of nuclei with unresolved point sources in the near-IR, the low soft X-ray to nuclear radio luminosity ratio (arguing against thermal emission powering the radio nuclei), and the lack of correlation between radio power and $\mathrm{H} \alpha$ luminosity, all support an origin of the detected radio nuclei in AGN related activity. This result is especially interesting for LINER ULIRGs for which signatures of AGNs have often been ambiguous in other wavebands. Such a high incidence of AGN would provide, for the first time, a large sample in which to study the interplay between AGN, starbursts, and galaxy mergers.
\end{abstract}

Key words. accretion, accretion disks - galaxies: active - galaxies: jets - radio continuum: galaxies - surveys

\section{Introduction}

The incidence of active galactic nuclei (AGN) and their relative importance, as compared to starbursts, in powering the far-IR emission of Ultraluminous Infrared Galaxies (ULIRGs; $L_{\mathrm{IR}} \geq 10^{12} L_{\odot}$ ) have been at the center of a long standing debate. The discovery of probable high $-z$ counterparts of ULIRGs - the dusty (presumably star forming) galaxies detected by SCUBA and MAMBO (e.g. Barger et al. 2000) gives the debate a new importance. There are now strong indications that the integrated light from the SCUBA sources and

Send offprint requests to: N. M. Nagar,

e-mail: nagar@astro.rug.nl

* Table 1 is also available in electronic form at the CDS via anonymous ftp to cdsarc.u-strasbg.fr $(130.79 .128 .5)$ or via http://cdsweb.u-strasbg.fr/cgi-bin/qcat?J/a+A/409/115
ULIRGs accounts for most or all of the sub-millimeter and farinfrared background (e.g. Cowie et al. 2002; Smail et al. 2002). Observational evidence that starbursts power ULIRGs includes the following: starbursts are present in almost every ULIRG, the well known correlation between radio and far infrared (FIR) luminosity observed in starbursts is followed (Yun et al. 2001), and large PAH equivalent widths (empirically related to starburst activity) are found in most ULIRGs investigated (Genzel et al. 1998; Lutz et al. 1998). The main evidence for AGNs in ULIRGs as a class comes from optical spectroscopy: roughly half of all ULIRGs show nuclear emission lines with ratios characteristic of Seyfert or LINER nuclei; of these half of the Seyfert type nuclei show broad $\left(\sim 5000-10000 \mathrm{~km} \mathrm{~s}^{-1}\right)$ permitted lines ( $\mathrm{H} \alpha$ or $\mathrm{Pa} \alpha$; Veilleux et al. 1999a,b). ULIRGs with "warm" (IRAS $25 \mu \mathrm{m}$ to $60 \mu \mathrm{m}$ flux ratio $>0.2$ ) infrared colors generally have Seyfert-like optical or near-infrared spectra, 
while hyperluminous $\left(L_{\mathrm{IR}} \geq 10^{13} L_{\odot}\right)$ objects all have warm infrared colors and most have Seyfert 2 spectra and/or "hidden" broad line regions (see e.g. Veilleux et al. 1995, 1999a,b, and the proceedings of the Ringberg conference on ULIRGs, 1999, Ap\&SS, vol. 266; hereafter Ringberg). The results listed above, and those from mid-IR spectroscopy (Rigopoulou et al. 1999; Tran et al. 2001) suggest a dividing line at $L_{\mathrm{IR}} \sim 10^{12.5} L_{\odot}$ with ULIRGs above this energetically dominated by AGN, and those below by starbursts. Direct observations of the AGN, such as its high brightness temperature radio nucleus and jets have been made in very few cases (see below). Hard X-ray observations, unfortunately available in only a handful of cases, can directly tell if an AGN (e.g. Mrk 231; Gallagher et al. 2002) or starburst (e.g. NGC 3256; Lira et al. 2002a), or both (e.g. NGC 6240; Lira et al. 2002b; Komossa et al. 2003) power the ULIRG.

ULIRGs are by nature dusty objects and their nuclei are expected to be heavily obscured in many cases. Detection of the nucleus itself is, therefore, best done at radio or hard X-ray wavebands, which are less affected by obscuration than the UV to near-IR. An advantage of the radio over the X-ray band lies in the much higher angular resolution available. A disadvantage of radio in comparison with X-ray observations is the negligible luminosity of the former; for this reason radio observations can never elucidate the dominant power source (starburst or AGN) in a ULIRG. Nevertheless, it is now clear that even lowluminosity accreting black holes have detectable compact radio cores (e.g. Nagar et al. 2002). These compact flat-spectrum radio cores are usually interpreted as the synchrotron selfabsorbed base of the jet which fuels larger-scale radio emission (Blandford \& Königl 1979; Falcke \& Biermann 1996). High resolution high frequency radio observations naturally pick out such AGN-related emission (compact and flat spectrum) while discriminating against starburst related emission (extended and steep-spectrum). Such a survey is therefore the most promising way to detect highly obscured accreting black holes embedded in the starforming environment of ULIRG nuclei. There has been no such large survey of ULIRGs so far. A few ULIRGs are known powerful radio sources, (e.g. Mrk 231 (Ulvestad et al. 1999) and 3C 273 (Mantovani et al. 2000)) and the AGNs in these objects have been well studied. Another four ULIRGs were studied by Smith et al. (1998a) as part of a sample of Luminous Infrared Galaxies (LIGs; $L_{\mathrm{IR}} \geq 10^{11} L_{\odot}$ ) and found to have radio cores with brightness temperature $\gtrsim 10^{6} \mathrm{~K}$, which is not high enough to claim unequivocally the presence of an AGN (see Condon et al. 1991). Of the 43 objects observed by Crawford et al. (1996) in the radio, only four are luminous enough to fit our definition of a ULIRG; one of these, $03521+0028$, is a member of the sample we study in this paper. A further 2 (of 7) ULIRGs were found to have $2.3 \mathrm{GHz}$ cores which are compact at 150 mas resolution above a detection limit of $\sim 5 \mathrm{mJy}$ in the survey of Seyfert ULIRGs by Roy et al. (1998). Despite their lower IR luminosities, about half of the LIGs studied in the above papers have a mas-scale radio core, a signature of an AGN. There is evidence that, with increasing IR luminosity, the AGN may increasingly dominate the energetics (Ringberg). Thus, one might expect a high incidence of dominant AGNs within a sample of ULIRGs.
We have, therefore, started a systematic high resolution radio survey of a well defined sample of ULIRGs. Here we present results of the first phase of the project: a $15 \mathrm{GHz}$ VLA survey. For consistency with Veilleux et al. (2002, hereafter VKS) we adopt $H_{0}=75 \mathrm{~km} \mathrm{~s}^{-1} \mathrm{Mpc}^{-1}$ and $q_{0}=0$ throughout this paper.

\section{Sample selection and observations}

As the parent sample, we use the complete flux-limited IRAS 1 Jy sample (Kim \& Sanders 1998) which consists of 118 ULIRGs drawn from the IRAS Faint Source Catalog. The selection criteria of Kim \& Sanders were $\delta>-40^{\circ}$, Galactic latitude $|b|>30^{\circ}, 60 \mu \mathrm{m}$ flux $f_{60}>1 \mathrm{Jy}, f_{60}>f_{12}$ (to exclude stars), warm IR colors $\left(\log \left(f_{60} / f_{100}\right)>-0.3\right)$, and $L_{\mathrm{IR}} \geq 10^{12} L_{\odot}$ (i.e. a ULIRG). The $1 \mathrm{Jy}$ sample is a reliable, complete collection of ULIRGs in the local universe, but is still small enough to be surveyed in a reasonable amount of time. The latest results on optical and IR imaging and spectroscopy of the sample as a whole can be found in Kim et al. (2002, hereafter KVS), VKS and references therein. All but one show signs of recent or ongoing interactions or mergers (VKS).

The subsample of the IRAS 1 Jy sample that we have observed in the radio, and report on here, comprises all 67 ULIRGs with $\delta>0^{\circ}$, and another 16 ULIRGs with $0^{\circ}>$ $\delta>-20^{\circ}$, as scheduling permitted. The median redshift of these 83 ULIRGs is $z_{\text {median }}=0.147$. In addition we observed two ULIRGs not in the IRAS 1 Jy sample: IRAS F00262+4251 from the "Genzel ULIRG sample" (Genzel et al. 1998) and IRAS F05246+0103 which was found to have a gigahertz peaked radio spectrum by Crawford et al. (1996).

The observations were made at $15 \mathrm{GHz}$ with the Very Large Array (VLA; Thompson et al. 1980) in A-configuration during a 24 hour run on 2002 February 22-23. Weather conditions were good and the atmospheric phase was stable through the whole run. Source positions used for the ULIRGs, taken from the near-IR positions listed in KVS, were expected to be accurate to $\lesssim 1^{\prime \prime}$. For each ULIRG with multiple nuclei, the phase center was chosen to be the average near-IR position of the nuclei and, in all cases, all nuclei fell within the effective field of view of the A-array at $15 \mathrm{GHz}$ (including bandwidth smearing and time averaging losses). For most sources, a five minute observation on source was sandwiched between two 1 min observations on a nearby phase calibrator. Only strong, "good" ("S" or "P"; see the calibration web page at www.nrao.edu) phase calibrators were used and the atmospheric variation was slow enough that the phase solutions for the phase calibrators could be reliably used for the sources in all cases. For the strongest ( $>0.5 \mathrm{Jy}$ ) target sources, phase referencing was not used. For optimal $u v$ coverage, we obtained two scans on each source separated by at least $1.5 \mathrm{hr}$ for all but four sources. For IRAS F04313-1649, IRAS F05246+0103, IRAS F20414-1651, and IRAS F21219-1757, only one scan on source was obtained. The sources were observed at elevations of $45^{\circ}-80^{\circ}$ except for those with $\delta<-10^{\circ}$ which had to be observed at elevations as low as $35^{\circ}$.

Standard calibration procedures within AIPS were followed, including antenna gain correction and atmospheric 
opacity correction. Variations of antenna gain with elevation were corrected by means of the latest antenna gain curves. A sky opacity of $1.5 \%$ was assumed, which is roughly what is expected at $15 \mathrm{GHz}$ in good weather conditions. Two four minute observations of $3 \mathrm{C} 286$ (made at elevations of $61^{\circ}$ and $74^{\circ}$ ) were used to set the flux density scale. Maps were made with the task IMAGR and, for sources stronger than about $3 \mathrm{mJy}$, iterative self-calibration was used to further improve the signal to noise in the map. The final noise in the maps is about $0.2 \mathrm{mJy}$ and the resolution is $\sim 150$ mas, equivalent to a linear resolution of $50 \mathrm{pc}$ to $800 \mathrm{pc}$ ( $420 \mathrm{pc}$ at the median redshift) for the ULIRGs observed here. Fluxes of detected sources were measured by fitting a single Gaussian using the task JMFIT. The highly accurate near-IR positions (KVS) made it easy to identify nuclear radio sources even at the $\sim 3 \sigma$ level.

\section{Results}

Our results are summarized in Table 1 . Column 1 gives the source name, Col. 2 the activity type, Cols. 3 and 4 the radio positions of the detected source(s) from our $15 \mathrm{GHz}$ observations, Col. 5 the offset between the $15 \mathrm{GHz}$ radio position and that of the near-infrared nucleus (KVS), Cols. 6 and 7 the peak and total flux densities, Col. 8 the redshift, Col. 9 the (assumed isotropic) monochromatic radio power and Col. 10 any comments.

\subsection{Detection statistics of 150 mas radio nuclei}

Our detection rate is high: radio sources are found towards 47 of 83 ULIRGs above a limit of $\sim 0.8 \mathrm{mJy}$. The nuclear origin of these radio sources is supported by the close (within the errors in most cases - Table 1, Col. 5) positional match to the corresponding near-IR nuclei. The detection rate is especially high for ULIRGs with AGN-like optical spectra: $\simeq 75 \%$ of all Seyferts and LINERs are detected in the radio (Fig. 1). The detection rate for ULIRGs with transitional H II + Seyfert/LINER spectra (see Table 1) is similarly high (6 of 10 nuclei). In contrast, the detection rate of ULIRGs with a pure $\mathrm{H}$ II type spectrum is significantly lower: only 2 of 15 or $14 \%$ (Fig. 1 and Table 1). Additionally, 3 of 8 ULIRGs with unknown nuclear spectral classification are detected in the radio. There is a large range in redshift among the radio-observed ULIRGs (Table 1); our non detection of radio cores in the Seyfert nuclei of the luminous $\left(M_{K}<-26.5 \mathrm{mag}\right)$ galaxies IRAS F11119+3257, IRAS F13218+0552, and IRAS F23498+2423 (see Fig. 2) may be due to them lying at the higher end of the redshift range of the sample (Table 1).

Given the above detection rates and the preference of AGNs to reside in "warm" ULIRGs (VKS), it is not surprising that the radio detection rate of warm ULIRGs (12 of 17) is higher than that of "cool" ULIRGs (35 of 66). However, with respect to the mid-IR ISO spectral classification (Lutz et al. 1999) we detect starburst ULIRGs (7 of 8) at the same rate as AGN ULIRGs ( 7 of 9). Both the above differences in detection rates are not reliable due to the small number of objects. The detectability of a radio nucleus is also related to the presence of an unresolved (w.r.t. the point spread function of the ground based images in

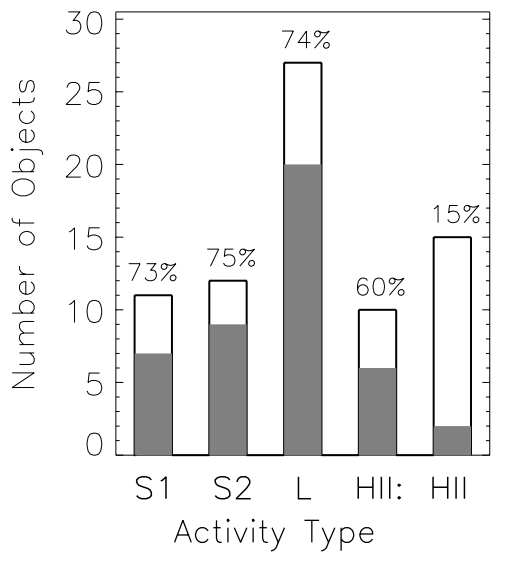

Fig. 1. Histogram showing the detection rates of $15 \mathrm{GHz}$ radio nuclei for ULIRGs with nuclear emission lines characteristic of (from left to right) Seyfert 1s, Seyfert 2s, LINERs, transitional Seyfert/LINER plus $\mathrm{H}$ II regions, and pure $\mathrm{H}$ II regions. The total and filled histograms show all radio-observed and all radio-detected ULIRGs, respectively. Activity type classifications are from Veilleux et al. (1999a). The radio detection rate is significantly higher among Seyferts and LINERs than among pure $\mathrm{H}$ II nuclei.

KVS and VKS) near-IR nucleus: 20 of 33 (61\%) nuclei with an unresolved near-IR nucleus are detected in the radio in contrast to only 10 of 53 (19\%) nuclei without unresolved nearIR nuclei. Further, the radio detectability is related to a higher $K^{\prime}$ luminosity of the nucleus in the central $4 \mathrm{kpc}$ (AGN plus galaxy; $M_{K^{\prime}}(4 \mathrm{kpc})$ ) but not to the total $K^{\prime}$ luminosity of the ULIRG (Fig. 2). The former dependence is significant at the 99.9\% level for all nuclei in the sample or for only the Seyfert and LINER nuclei. No dependence of radio source detectability could be found on any of the following: interaction class (an estimate of the merger or interaction stage as defined in VKS), galaxy morphology, or $R$ or $K^{\prime}$ absolute magnitude of the total ULIRG.

Most of the detected radio cores are compact. Our observations are not expected to detect extended emission given the limited signal to noise ratio of most detections and the "snapshot" nature of the $u v$ coverage. The two clear exceptions are IRAS F15327+2340 (Arp 220) which is discussed below and IRAS F23389+0300 (4C 03.60) which has twin slightly resolved radio "lobes" (Fig. 3) separated by 0.3 (830 pc at $D=620 \mathrm{Mpc}$ ). A comparison with lower resolution fluxes at other frequencies (e.g. the $1.4 \mathrm{GHz}$ flux from the FIRST VLA radio survey; White et al. 1997) shows that these "lobes" probably have a steep spectrum. The optical and near-IR images of $4 \mathrm{C} 03.60$ show it to have two nuclei separated by $\sim 2^{\prime \prime}$, and the radio lobes are centered on a point close to the northern near-IR and optical nucleus (Fig. 3).

It is interesting to examine the radio source detections in ULIRGs with multiple optical or IR components. Of the radio-detected ULIRGs, VKS (using ground based near-IR images) have classified 18 as having two components and 3 as having three components. We find no dependence of radio source detectability on nuclear separation between the multiple near-IR nuclei. In 19 of these 21 ULIRGs, only one of the two or three near-IR nuclei is detected in our radio 
Table 1. New $2 \mathrm{~cm}$ VLA observations of the IRAS 1 Jy ULIRG sample.

\begin{tabular}{|c|c|c|c|c|c|c|c|c|c|}
\hline $\begin{array}{l}\text { IRAS } \\
\text { FSC }\end{array}$ & $\begin{array}{l}\text { Activity } \\
\text { Type }\end{array}$ & $\begin{array}{c}\text { RA } \\
(\mathrm{J} 2000)\end{array}$ & $\begin{array}{c}\text { Dec } \\
(\mathrm{J} 2000)\end{array}$ & $\begin{array}{r}\Delta \\
\left({ }^{\prime \prime}\right)\end{array}$ & $\begin{array}{r}S_{\text {peak }} \\
(\mathrm{mJy} / \\
\text { beam })\end{array}$ & $\begin{array}{r}S_{\text {tot }} \\
(\mathrm{mJy})\end{array}$ & $\bar{z}$ & $\begin{array}{r}\log P_{15 \mathrm{GHz}}^{\text {core }} \\
\left(\mathrm{W} \mathrm{Hz}{ }^{-1}\right)\end{array}$ & Com. \\
\hline (1) & (2) & (3) & (4) & (5) & (6) & (7) & (8) & (9) & (10) \\
\hline $00091-0738 \mathrm{~S}$ & H2: & 001143.273 & -072207.34 & 0.49 & 1.1 & 1.1 & 0.118 & 22.49 & \\
\hline $00188-0856$ & $\mathrm{~L}$ & 002126.513 & -083926.01 & 1.19 & 1.2 & 2.1 & 0.128 & 22.84 & \\
\hline 00397-1312 & $\mathrm{H} 2$ & & & & & $<0.8$ & 0.261 & $<23.07$ & \\
\hline 01166-0844 & $\mathrm{H} 2$ : & & & & & $<0.8$ & 0.118 & $<22.35$ & \\
\hline 01298-0744 & $\mathrm{H} 2$ : & 013221.413 & -072908.34 & 0.16 & 2.1 & 2.5 & 0.136 & 22.97 & \\
\hline $01355-1814$ & $\mathrm{H} 2$ & & & & & $<0.8$ & 0.192 & $<22.79$ & \\
\hline 01494-1845 & & & & & & $<0.8$ & 0.158 & $<22.61$ & \\
\hline $01572+0009$ & $\mathrm{~S} 1$ & 015950.253 & 002340.86 & 0.49 & 1.6 & 1.6 & 0.163 & 22.94 & \\
\hline $02021-2103$ & & & & & & $<0.8$ & 0.116 & $<22.34$ & \\
\hline $02411+0353$ & $\mathrm{H} 2$ & & & & & $<0.8$ & 0.144 & $<22.53$ & \\
\hline 03209-0806 & $\mathrm{H} 2$ : & & & & & $<0.8$ & 0.166 & $<22.66$ & \\
\hline $03250+1606$ & $\mathrm{~L}$ & & & & & $<0.8$ & 0.129 & $<22.43$ & \\
\hline Z03521+0028 E & $\mathrm{L}$ & 035442.277 & 003703.24 & 1.30 & 1.4 & 1.6 & 0.152 & 22.88 & \\
\hline $04313-1649$ & & & & & & $<0.8$ & 0.268 & $<23.09$ & \\
\hline 05024-1941 & $\mathrm{S} 2$ & 050436.563 & -193702.92 & 1.64 & 3.1 & 3.4 & 0.192 & 23.42 & \\
\hline $07599+6508$ & $\mathrm{~S} 1$ & 080430.472 & 645952.76 & 0.57 & 2.4 & 2.9 & 0.149 & 23.12 & \\
\hline $08201+2801$ & $\mathrm{H} 2$ & & & & & $<0.8$ & 0.168 & $<22.67$ & \\
\hline $08474+1813$ & & 085018.332 & 180201.17 & 0.31 & 0.6 & 1.4 & 0.145 & 22.78 & \\
\hline $08559+1053$ & $\mathrm{~S} 2$ & & & & & $<0.8$ & 0.149 & $<22.56$ & \\
\hline $08572+3915 \mathrm{NW}$ & $\mathrm{L}:$ & 090025.376 & 390354.15 & 1.14 & 2.7 & 3.3 & 0.058 & 22.34 & \\
\hline $08591+5248$ & & & & & & $<0.8$ & 0.158 & $<22.61$ & \\
\hline $09039+0503$ & $\mathrm{~L}$ & & & & & $<0.8$ & 0.125 & $<22.40$ & \\
\hline $09116+0334 \mathrm{E}$ & $\mathrm{L}:$ & 091413.794 & 032201.36 & 0.21 & 2.3 & 2.6 & 0.146 & 23.05 & \\
\hline $09463+8141$ & $\mathrm{~L}$ & 095300.204 & 812728.42 & 0.33 & 0.5 & 1.7 & 0.156 & 22.93 & \\
\hline $09539+0857$ & $\mathrm{~L}$ & 095634.333 & 084306.31 & 0.75 & 1.2 & 1.2 & 0.129 & 22.61 & \\
\hline $10035+2740$ & & & & & & $<0.8$ & 0.165 & $<22.65$ & \\
\hline $10091+4704$ & L: & 101216.766 & 464943.63 & 0.77 & 0.7 & 1.0 & 0.246 & 23.11 & \\
\hline $10190+1322 \mathrm{E}$ & $\mathrm{H} 2$ & 102142.754 & 130655.63 & 1.02 & 0.5 & 0.5 & 0.077 & 21.77 & \\
\hline $10378+1108$ & $\mathrm{~L}$ & 104029.169 & 105318.14 & 0.45 & 1.0 & 1.2 & 0.136 & 22.66 & \\
\hline $10494+4424$ & $\mathrm{~L}$ & 105223.564 & 440846.95 & 0.52 & 1.1 & 2.8 & 0.092 & 22.68 & \\
\hline $10594+3818$ & $\mathrm{H} 2$ & & & & & $<0.8$ & 0.158 & $<22.61$ & \\
\hline $11028+3130$ & $\mathrm{~L}$ & 110537.533 & 311432.10 & 1.55 & 0.7 & 0.8 & 0.199 & 22.82 & \\
\hline $11119+3257$ & $\mathrm{~S} 1$ & & & & & $<0.8$ & 0.189 & $<22.77$ & \\
\hline $11180+1623 \mathrm{~W}$ & L: & 112041.173 & 160656.42 & 1.04 & 0.9 & 0.9 & 0.166 & 22.73 & \\
\hline $11387+4116$ & $\mathrm{H} 2$ : & & & & & $<0.8$ & 0.149 & $<22.56$ & \\
\hline $11506+1331$ & $\mathrm{H} 2$ : & 115314.233 & 131427.73 & 1.33 & 0.4 & 1.6 & 0.127 & 22.72 & \\
\hline $11582+3020$ & $\mathrm{~L}$ & & & & & $<0.8$ & 0.223 & $<22.92$ & \\
\hline $12018+1941$ & & 120424.543 & 192509.73 & 0.21 & 0.6 & 1.1 & 0.168 & 22.81 & \\
\hline $12032+1707 \mathrm{~N}$ & $\mathrm{~L}$ & 120547.722 & 165108.27 & 0.12 & 1.1 & 1.9 & 0.217 & 23.27 & \\
\hline $12112+0305 \mathrm{NE}$ & $\mathrm{L}$ & 121346.057 & 024841.53 & 0.51 & 3.3 & 3.9 & 0.073 & 22.61 & 3 \\
\hline $12265+0219$ & $\mathrm{~S} 1$ & 122906.700 & 020308.60 & 0.65 & 21427.0 & 21614.0 & 0.159 & 27.05 & 1,5 \\
\hline $12447+3721$ & $\mathrm{H} 2$ & & & & & $<0.8$ & 0.158 & $<22.61$ & \\
\hline $12540+5708$ & $\mathrm{~S} 1$ & 125614.234 & 565225.24 & 0.47 & 193.5 & 211.5 & 0.042 & 23.86 & 4 \\
\hline $13218+0552$ & $\mathrm{~S} 1$ & & & & & $<0.8$ & 0.205 & $<22.85$ & \\
\hline $13342+3932$ & $\mathrm{~S} 1$ & & & & & $<0.8$ & 0.179 & $<22.73$ & \\
\hline $13428+5608$ & $\mathrm{~S} 2$ & 134442.129 & 555313.51 & 1.07 & 6.7 & 18.8 & 0.037 & 22.70 & 3 \\
\hline $13443+0802 \mathrm{NE}$ & $\mathrm{H} 2$ & 134651.094 & 074718.84 & 0.60 & 0.9 & 1.3 & 0.135 & 22.68 & \\
\hline $13451+1232 \mathrm{~W}$ & S1: & 134733.362 & 121724.24 & 1.20 & 1320.0 & 1475.0 & 0.122 & 25.65 & $1,5,6,7$ \\
\hline $13469+5833$ & $\mathrm{H} 2$ & & & & & $<0.8$ & 0.158 & $<22.61$ & \\
\hline $13509+0442$ & $\mathrm{H} 2$ & & & & & $<0.8$ & 0.136 & $<22.48$ & \\
\hline $13539+2920 \mathrm{NW}$ & $\mathrm{H} 2$ : & 135609.990 & 290535.13 & 0.13 & 0.7 & 3.1 & 0.108 & 22.86 & \\
\hline $13539+2920 \mathrm{SE}$ & $\mathrm{H} 2$ : & 135610.310 & 290534.18 & 0.16 & & 0.9 & 0.108 & 22.32 & 2 \\
\hline $14060+2919$ & $\mathrm{H} 2$ & & & & & $<0.8$ & 0.117 & $<22.34$ & \\
\hline $14070+0525$ & $\mathrm{~S} 2$ & 140931.245 & 051131.51 & 0.52 & 0.7 & 0.7 & 0.265 & 23.02 & \\
\hline $14197+0813$ & & 142211.801 & 075925.80 & 1.75 & & 0.6 & 0.131 & 22.36 & \\
\hline
\end{tabular}


Table 1. continued.

\begin{tabular}{|c|c|c|c|c|c|c|c|c|c|}
\hline $\begin{array}{l}\text { IRAS } \\
\text { FSC }\end{array}$ & $\begin{array}{l}\text { Activity } \\
\text { Type }\end{array}$ & $\begin{array}{c}\text { RA } \\
(\mathrm{J} 2000)\end{array}$ & $\begin{array}{c}\text { Dec } \\
(\mathrm{J} 2000)\end{array}$ & $\begin{array}{r}\Delta \\
\left({ }^{\prime \prime \prime}\right)\end{array}$ & $\begin{array}{r}S_{\text {peak }} \\
(\mathrm{mJy} / \\
\text { beam })\end{array}$ & $\begin{array}{r}\begin{array}{r}S_{\text {tot }} \\
(\mathrm{mJy})\end{array} \\
\end{array}$ & $\bar{z}$ & $\begin{array}{r}\log P_{15 \mathrm{GHz}}^{\text {core }} \\
\left.(\mathrm{W} \mathrm{Hz})^{-1}\right)\end{array}$ & Com. \\
\hline (1) & (2) & (3) & (4) & (5) & (6) & (7) & (8) & (9) & (10) \\
\hline $14202+2615$ & $\mathrm{H} 2$ & & & & & $<0.8$ & 0.159 & $<22.62$ & \\
\hline $14394+5332 \mathrm{EW}$ & S2 & 144104.396 & 532008.78 & 0.71 & 5.1 & 5.6 & 0.105 & 23.09 & \\
\hline $15001+1433 \mathrm{E}$ & S2 & 150231.924 & 142135.26 & 0.25 & 2.8 & 3.3 & 0.162 & 23.25 & \\
\hline $15043+5754 \mathrm{~S}$ & $\mathrm{H} 2$ & 150539.457 & 574306.59 & 0.80 & 0.7 & 1.3 & 0.151 & 22.78 & \\
\hline $15206+3342$ & $\mathrm{H} 2$ & & & & & $<0.8$ & 0.125 & $<22.40$ & \\
\hline $15225+2350$ & $\mathrm{H} 2$ : & 152443.870 & 234010.29 & 0.95 & 1.3 & 1.3 & 0.139 & 22.71 & \\
\hline $15327+2340$ & $\mathrm{~L}$ & 153457.291 & 233011.33 & 1.08 & 17.5 & 87.2 & 0.018 & 22.74 & 3 \\
\hline $16156+0146 \mathrm{NW}$ & S2 & 161809.364 & 013921.76 & 0.42 & 3.5 & 3.5 & 0.132 & 23.09 & \\
\hline $16300+1558$ & $\mathrm{~L}$ & & & & & $<0.8$ & 0.242 & $<23.00$ & \\
\hline $16333+4630 \mathrm{~W}$ & $\mathrm{~L}$ & 163452.349 & 462452.63 & 0.43 & 1.0 & 2.3 & 0.191 & 23.24 & \\
\hline $16468+5200$ & $\mathrm{~L}$ & & & & & $<0.8$ & 0.150 & $<22.57$ & \\
\hline $16474+3430$ & $\mathrm{H} 2$ : & & & & & $<0.8$ & 0.111 & $<22.30$ & \\
\hline $16487+5447 \mathrm{~W}$ & $\mathrm{~L}:$ & 164946.895 & 544235.33 & 0.19 & 2.0 & 2.8 & 0.104 & 22.78 & \\
\hline $17028+5817 \mathrm{~W}$ & $\mathrm{~L}:$ & 170341.927 & 581344.62 & 0.25 & 1.0 & 1.1 & 0.106 & 22.40 & \\
\hline $17044+6720$ & $\mathrm{~L}$ & 170428.450 & 671628.21 & 0.36 & 2.4 & 1.9 & 0.135 & 22.85 & \\
\hline $17068+4027$ & $\mathrm{H} 2$ & & & & & $<0.8$ & 0.179 & $<22.73$ & \\
\hline $17179+5444$ & S2 & 171854.392 & 544148.27 & 0.45 & 24.0 & 25.8 & 0.147 & 24.06 & \\
\hline $20414-1651$ & $\mathrm{H} 2$ : & 204418.160 & -164016.83 & 0.53 & 3.1 & 4.5 & 0.086 & 22.82 & \\
\hline $21208-0519$ & $\mathrm{H} 2$ & & & & & $<0.8$ & 0.130 & $<22.44$ & \\
\hline $21219-1757$ & S1 & 212441.641 & -174445.89 & 0.30 & 1.8 & 1.8 & 0.113 & 22.67 & \\
\hline $21477+0502$ & $\mathrm{~L}:$ & & & & & $<0.8$ & 0.171 & $<22.68$ & \\
\hline $22541+0833$ & S2 & & & & & $<0.8$ & 0.166 & $<22.66$ & \\
\hline $23060+0505$ & S1: & 230833.954 & 052129.76 & 0.52 & 1.5 & 2.4 & 0.173 & 23.17 & 6 \\
\hline $23129+2548$ & L: & 231521.402 & 260432.41 & 0.22 & 3.1 & 3.2 & 0.179 & 23.33 & \\
\hline $23233+2817$ & $\mathrm{~S} 2$ & & & & & $<0.8$ & 0.114 & $<22.32$ & \\
\hline $23234+0946 \mathrm{~W}$ & $\mathrm{~L}$ & 232556.143 & 100249.52 & 1.38 & 0.9 & 2.4 & 0.128 & 22.90 & \\
\hline $23327+2913$ & L: & & & & & $<0.8$ & 0.107 & $<22.27$ & \\
\hline $23389+0300 \mathrm{~N}$ & S2 & 234130.323 & 031726.72 & 0.27 & 12.4 & 40.9 & 0.145 & 24.24 & 5 \\
\hline $23498+2423$ & S1: & & & & & $<0.8$ & 0.212 & $<22.88$ & 6 \\
\hline
\end{tabular}

Columns are: (1) ULIRG name from the IRAS faint source catalog, to be preceeded by "IRAS $F$ ". When the radio source is clearly identifiable with one subcomponent of a multi-component ULIRG then the component (following the naming convention of KVS) is also listed; (2) nuclear activity type as given by Veilleux et al. (1999a). "L" represents LINER, "S" represents Seyfert, "H" represents an H II region type spectrum. "2" implies that no broad H $\alpha$ or broad Pa $\alpha$ is detected, and "1.0" implies that broad $\mathrm{H} \alpha$ and/or broad Pa $\alpha$ are detected (Veilleux et al. 1999a, 1999b). The ":” symbol represents an uncertain classification. Nuclei classified as "H II:" display spectra which are transitional between H II regions and Seyferts/LINERs; (3) and (4) $15 \mathrm{GHz}$ radio position, from our maps; (5) offset, in arcsec, between the $15 \mathrm{GHz}$ radio position and the position of the near-IR nucleus as listed in KVS; (6) and (7) peak flux-density and total flux, obtained by fitting a single Gaussian (with peak flux-density, major and minor axes as free parameters) to the radio nuclear source in our maps; (8) redshift of the ULIRG, as listed in Veilleux et al. (1999); (9) the logarithm of the core radio power (derived from the total radio flux as listed in Col. 7); (10) Comments as listed below.

Comments: (1) phase-calibration not used; the radio position listed is taken from the VLA calibrator database; (2) uncertain detection. Flux measured by summing within an aperture rather than by a Gaussian fit; (3) known to have a milli-arcsec scale radio core (Smith et al. 1998); (4) known to have a milli-arcsec scale radio core (Ulvestad et al. 1998); (5) known to have a milli-arcsec scale radio core; (6) broad $\mathrm{Pa} \alpha$ (but not broad $\mathrm{H} \alpha$ ) is detected in this object, so it is counted as a Seyfert 1 in this paper; (7) the highly accurate radio position (Ma et al. 1998) is offset from both near-IR nuclei, but is closer to the "W" nucleus.

observations. The remaining two ULIRGs are worth remarking on here. Arp 220 has two well known (Smith et al. 1998) extended radio nuclei with separation $\sim 0$ ' 96 , corresponding to the two near-IR nuclei. The exact registration between radio and near-IR nuclei is discussed in Scoville et al. (1998). In IRAS F13539+2920 we have potentially detected radio sources toward both near-IR nuclei though the detection of the SE nucleus is not reliable. The radio source in most ( 15 out of 21 ) of the radio-detected ULIRGs with multiple near-IR nuclei coincides with the near-IR nucleus with the brighter
$M_{K^{\prime}}(4 \mathrm{kpc})$. In three other cases, the multiple nuclei have similar (to within $\sim 0.2 \mathrm{mag}$ ) $M_{K^{\prime}}(4 \mathrm{kpc})$. Only in the three remaining cases are the radio-detected nuclei the fainter (w.r.t. the other nuclei in the ULIRG) in $M_{K^{\prime}}(4 \mathrm{kpc})$ : IRAS F03521+0028 (1 mag fainter in $M_{K^{\prime}}(4 \mathrm{kpc})$ though only 0.1 mag fainter in $M_{R}(4 \mathrm{kpc})$ ), IRAS F09116+0334 (this is an unusual case as the radio source is not towards the bright Seyfert galaxy but instead towards the visually fainter but more compact absorption line galaxy), and IRAS F11180+1623 (fainter in $M_{K^{\prime}}(4 \mathrm{kpc}$ ) but more compact). 

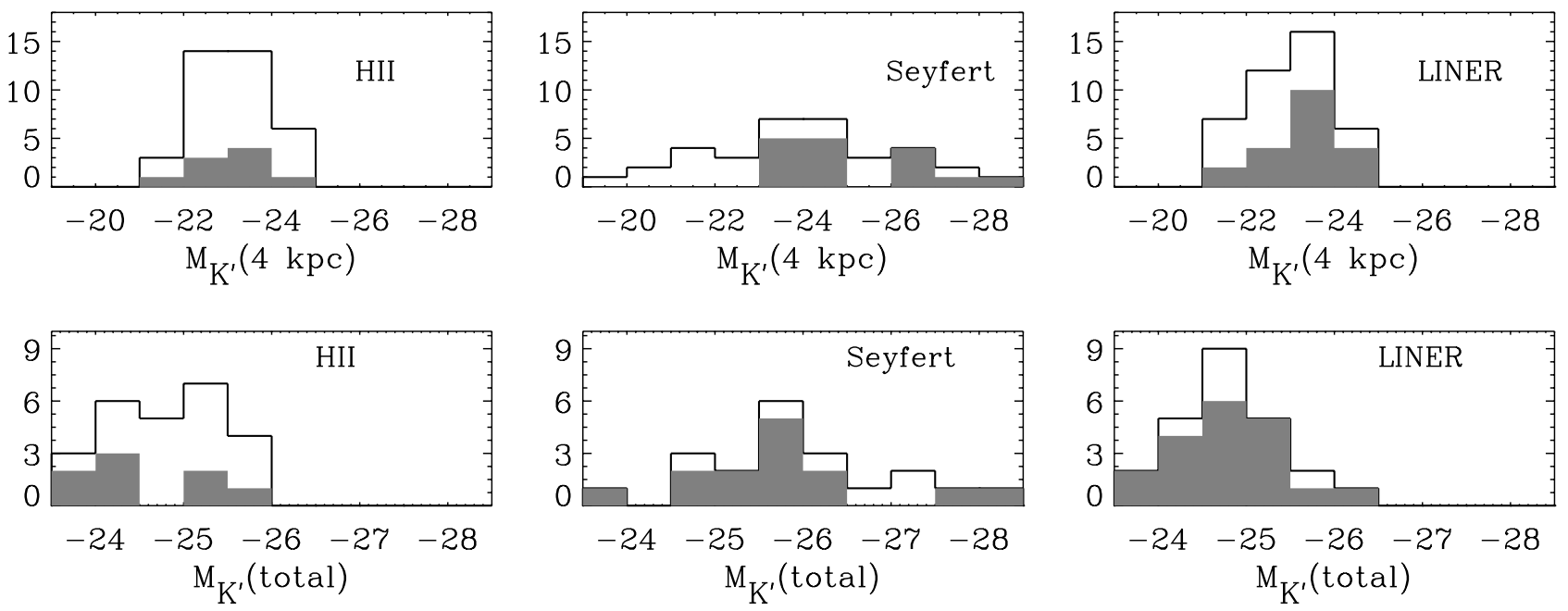

Fig. 2. Histograms of (top) absolute magnitude of the inner $4 \mathrm{kpc}$ of the ULIRG component in the $K^{\prime}$ band (i.e. AGN + galaxy), when available; (bottom) absolute magnitude of the (total) ULIRG in the $K^{\prime}$ band. Data are from VSK and KVS. The total and filled histograms represent all ULIRGs (or all ULIRG components) observed and detected in the radio by us, respectively. Note that the horizontal scales are different between top and bottom panels. The radio detection rate increases with increasing nuclear (AGN plus central $4 \mathrm{kpc}$ of nucleus) $K^{\prime}$-band luminosity but is independent of the $K^{\prime}$ luminosity of the total ULIRG.

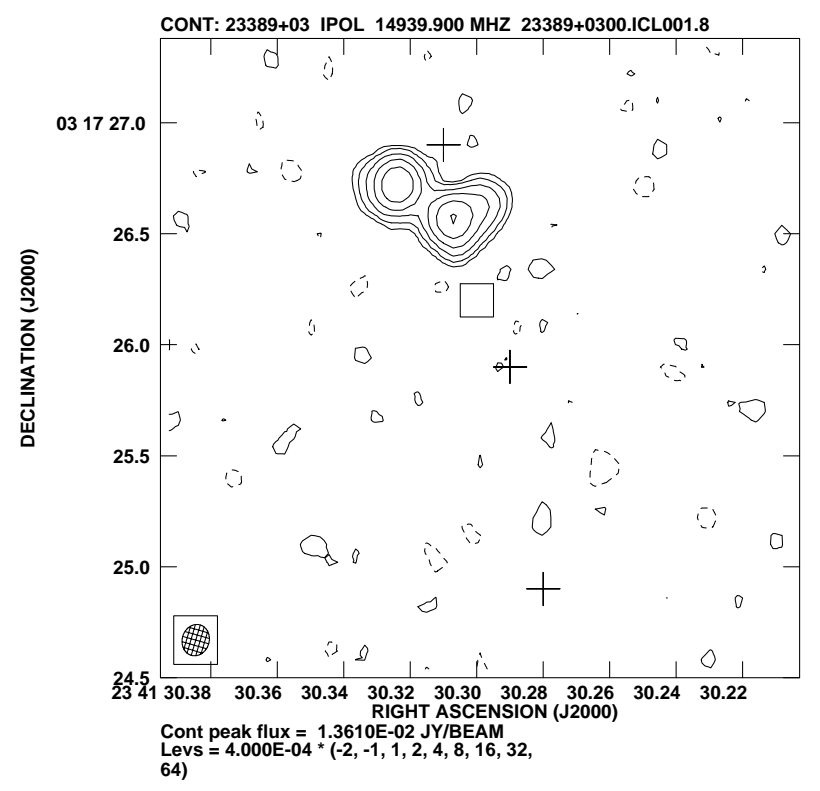

Fig. 3. $15 \mathrm{GHz}(2 \mathrm{~cm})$ VLA map of IRAS F23389+0300 (4C 03.60). The crosses mark the positions of the two near-IR nuclei and their average position, and the rectangle marks the position of the northern optical nucleus (KVS). The southern optical nucleus lies off the bottom of the figure.

\subsection{Are the radio sources $A G N$ related?}

At the resolution of the observations presented here only three nuclei, all well known AGNs, have radio brightness temperatures $>10^{6} \mathrm{~K}$. The other detections have typical brightness temperature limits $T_{\mathrm{b}}>10^{2.5-3.5} \mathrm{~K}$, much lower than the limit (at least $10^{6} \mathrm{~K}$; Condon et al. 1991) required to confidently invoke an AGN instead of a highly absorbed nuclear starburst. Another potential source of the radio emission is radio supernova(e) (RSN; Weiler et al. 2002) whose high brightness temperature can mimic an AGN in our observations. It is therefore relevant to explore whether the compact radio cores we detect trace AGN, nuclear starburst activity, or radio supernovae.

A comparison of nuclear radio fluxes with the soft $(0.5-$ $2 \mathrm{keV}$ ) X-ray emission from ULIRGs is a powerful test of whether both originate in thermal bremsstrahlung. The median nuclear monochromatic luminosity of the radio-detected ULIRGs is $L_{15 \mathrm{GHz}}$ (median) $10^{30} \mathrm{erg} \mathrm{s}^{-1} \mathrm{~Hz}^{-1}$, so (following the argument outlined in Falcke et al. 2000) thermal bremsstrahlung would imply $0.5-2 \mathrm{keV}$ luminosities around $\sim 10^{46} \mathrm{erg} \mathrm{s}^{-1}$, significantly higher than observed in ULIRGs (Boller 1999; Braito et al. 2002). High column densities could absorb the nuclear soft X-rays but the detection of broad $\mathrm{H} \alpha$ in several of the sample argues against such high columns at least in the Seyfert 1s. Also, the high frequency we used is more biased towards detecting flat or inverted spectrum sources (typical of AGN nuclei) rather than extended steep spectrum sources (common among starbursts) as illustrated by our previous results with low-luminosity AGN (Nagar et al. 2002). The compactness of the detected radio nuclei ( $\leq 150$ mas or typically $\leq 50-800 \mathrm{pc}$ for the ULIRGs observed) also argues against extended starbursts. Another argument for the radio nuclei being AGN related (rather than starburst or RSN) is the preferential detection of Seyfert and LINER ULIRGs as opposed to H II nuclei ULIRGs even though both subgroups have similar median FIR luminosities. Also, no clear correlation is seen between the nuclear $\left(\sim 2^{\prime \prime} \times 2^{\prime \prime}\right.$ aperture) $\mathrm{H} \alpha$ luminosity and the radio power of the nuclei, arguing against both originating in the same source.

It is notable that Arp 220, with its well known starburst dominated nuclei, was detected as extended in our survey, and thus the radio nuclei (which originate in at least $15 \mathrm{RSN}$; Smith et al. 1998, 1999) were not mistaken as AGN-related. 
The radio-detected ULIRGs in our survey have $z_{\text {median }}=0.136$ and $P_{\text {median }}^{2 \mathrm{~cm}}=22.84$ Watt $\mathrm{Hz}^{-1}$; powering such a "median" ULIRG would require $\gtrsim 10$ RSN (Weiler et al. 2002) all at peak radio power or $\gtrsim 18$ of the typical RSN found in Arp 220.

It is worth emphasizing the importance of obtaining deeper radio observations in order to detect the other nuclei in ULIRGs with multiple near-IR nuclei. VKS have shown that the luminosity ratio of the brightest to faintest component in multiple component ULIRGs is typically less than 4. The preferential radio detection of the more luminous near-IR nuclei thus leads us to believe that radio observations a factor of a few deeper should be able to detect multiple nuclei in several of the ULIRG systems. Milli-arcsec observations of the VLAdetected ULIRGs can both confirm their AGN nature and perhaps find binary massive black hole systems at $<150$ mas separations. Our ongoing followup VLBI observational campaign will attempt to do this.

\section{Conclusions}

We have observed 83 of the 118 ULIRGs in the IRAS 1 Jy sample at $15 \mathrm{GHz}$ with the VLA at a resolution of 150 mas and a flux limit of $\sim 0.8 \mathrm{mJy}$. Our detection rate of nuclear radio cores is high: about $75 \%$ of all LINER and Seyfert type ULIRGs are detected. The detection rate is significantly lower (only 2 of 15 or $14 \%$ ) for ULIRGs with a pure H II nuclear spectrum. For multiple component ULIRGs only one (if any) nucleus - typically the brighter near-IR one - was detected in our snapshot observations. Deeper observations to detect binary or multiple AGN are highly desirable. Several factors argue for an origin of the radio emission in AGN rather than starburst-related activity or radio supernovae. These are: the compactness and high power of the radio nuclei, the preferential detection of AGNtype nuclei than $\mathrm{H}$ II nuclei, the preferential detection of nuclei with unresolved point sources in the near-IR, the low soft X-ray to nuclear radio luminosity ratio (arguing against a thermal origin for the radio emission), and the lack of correlation between radio power and $\mathrm{H} \alpha$ luminosity. Such a high incidence of AGN in ULIRGs opens an avenue to study the inter-relationships between starbursts, AGNs and the galaxy merging process.

Acknowledgements. This work was partially supported by the Italian Ministry for University and Research (MURST) under grant Cofin0002-36 and the Italian Space Agency (ASI) under grant 1/R/27/00. This research was supported in part by NASA through grant NAG81755 to the University of Maryland. NN thanks the Raman Research Institute for hospitality during the writing of a part of this paper. SV thanks the California Institute of Technology and the Observatories of the Carnegie Institution for their hospitality.

\section{References}

Barger, A. J., Cowie, L. L., \& Richards, E. A. 2000, AJ, 119, 2092 Blandford, R. D., \& Königl, A. 1979, ApJ, 232, 34

Boller, T. 1999, Ap\&SS, 266, 49
Braito, V., Franceschini, A., Della Ceca, R., et al. 2002, New Visions of the X-ray Universe in the XMM-Newton and Chandra Era, ESA-SP, ed. F. Jansen [astro-ph/0202352]

Condon, J. J., Cotton, W. D., Greisen, E. W., et al. 1998, AJ, 115, 1693

Condon, J. J., Huang, Z.-P., Yin, Q. F., \& Thuan, T. X. 1991, ApJ, 378, 65

Cowie, L. L., Barger, A. J., \& Kneib, J.-P. 2002, AJ, 123, 2197

Crawford, T., Marr, J., Partridge, B., \& Strauss, M. A. 1996, ApJ, 460, 225

Falcke, H., \& Biermann, P. L. 1996, A\&A, 308, 321

Falcke, H., Nagar, N. M., Wilson, A. S., \& Ulvestad, J. S. 2000, ApJ, 542, 197

Gallagher, S. C., Brandt, W. N., Chartas, G., Garmire, G. P., \& Sambruna, R. M. 2002, ApJ, 569, 655

Genzel, R., Lutz, D., Shurm, E., et al. 1998, ApJ, 498, 579

Kim, D.-C., \& Sanders, D. B. 1998, ApJS, 119, 41

Kim, D.-C., Veilleux, S., \& Sanders, D. B. 2002, ApJS, 143, 277 (KVS)

Komossa, S., Burwitz, V., Hasinger, G., et al. 2003, ApJ, 582, L15

Lavalley, M., Isobe, T., \& Feigelson, E. 1992, in Astronomical Data Analysis Software and Systems I, ed. D. Worrall, C. Biemesderfer \& J. Barnes (San Francisco: ASP), 25, 245 (ASURV)

Lira, P., Ward, M., Zezas, A., Alonso-Herrero, A., \& Ueno, S. 2002a, MNRAS, 330, 259

Lira, P., Ward, M. J., Zezas, A., \& Murray, S. S. 2002b, MNRAS, 333, 709

Lutz, D., Spoon, H. W. W., Rigopoulou, D., Moorwood, A. F. M., \& Genzel, R. 1998, ApJ, 505, L103

Lutz, D., Veilleux, S., \& Genzel, R. 1999, ApJ, 517, L13

Ma, C., Arias, E. F., Eubarks, T. M., et al. 1998, AJ, 116, 516

Mantovani, F., Junor, W., McHardy, I. M., \& Valerio, C. 2000, A\&A, 354,497

Nagar, N. M., Falcke, H., Wilson, A. S., \& Ulvestad, J. S. 2002, A\&A, 392, 53

Rigopoulou, D., Spoon, H. W. W., Genzel, R., et al. 1999, AJ, 118, 2625

Roy, A. L., Norris, R. P., Kesteven, M. J., Troup, E. R., \& Reynolds, J. E. 1998, MNRAS, 301, 1019

Scoville, N. Z., Evans, A. S., Dinshaw, N., et al. 1998, ApJ, 492, L107 Smail, I., Ivison, R. J., Blain, A. W., \& Kneib, J.-P. 2002, MNRAS, 331, 495

Smith, H. E., Lonsdale, C. J., \& Lonsdale, C. J. 1998a, ApJ, 492, 137

Smith, H. E., Lonsdale, C. J., Lonsdale, C. J., \& Diamond, P. J. 1998b, ApJ, 493, L17

Smith, H. E., Lonsdale, C. J., Lonsdale, C. J., \& Diamond, P. J. 1999, Ap\&SS, 266, 125

Thompson, A. R., Clark, B. G., Wade C. M., \& Napier, P. J. 1980, ApJS, 44, 151

Tran, Q. D., Lutz, D., Genzel, R., et al. 2001, ApJ, 552, 527

Ulvestad, J. S., Wrobel, J. M., Roy, A. L., et al. 1999, ApJ, 517, L81

Veilleux, S., Kim, D.-C., \& Sanders, D. B. 1999a, ApJ, 522, 113

Veilleux, S., Kim, D.-C., \& Sanders, D. B. 2002, ApJS, 143, 315 (VKS)

Veilleux, S., Kim, D.-C., Sanders, D. B., Mazzarella, J. M., \& Soifer, B. T. 1995, ApJS, 98,171

Veilleux, S., Sanders, D. B., \& Kim, D.-C. 1999b, ApJ, 522, 139

Weiler, K. W., Panagia, N., Montes, M. J., \& Sramek, R. A. 2002, ARA\&A, 40, 387

White, R. L., Becker, R. H., Helfand, D. J., \& Gregg, M. D. 1997, ApJ, 475, 479 (FIRST)

Yun, M. S., Reddy, N. A., \& Condon, J. J. 2001, ApJ, 554, 803 